\title{
A Phase II Study of Sequential Treatment with Anthracycline and Taxane Followed by Eribulin in Patients with HER2-negative, Locally Advanced Breast Cancer (JBCRG-17)
}

Ippei Fukada ( $\square$ ippei.fukada@jfcr.or.jp )

Cancer institute Hospital https://orcid.org/0000-0001-7272-3708

Yoshinori Ito

Cancer Institute Hospital

Naoto Kondo

Nagoya City University: Nagoya Shiritsu Daigaku

Shoichiro Ohtani

Hiroshima City Hiroshima Citizens Hospital

Masaya Hattori

Aichi Cancer Center: Aichi-ken Gan Center

Eriko Tokunaga

National Kyushu Cancer Center: Kyushu Gan Center

Nobuki Matusnami

Osaka Rosai Hospital: Osaka Rosai Byoin

Kohjiro Mashino

Oita Prefectural Hospital: Oita Kenritsu Byoin

Taijiro Kosaka

Juntendo University: Juntendo Daigaku

Masahiko Tanabe

Juntendo University: Juntendo Daigaku

Daisuke Yotusmoto

Sagara Hospital

Kosho Yamanouchi

Nagasaki University Graduate School of Biomedical Sciences

Masataka Sawaki

Aichi Cancer Center: Aichi-ken Gan Center

Masahiro Kashiwaba

Breastopia Miyazaki Hospital

Hidetaka Kawabata

Toranomon Hospital: Toranomon Byoin 


\section{Katsumasa Kuroi}

Tokyo Metropolitan Cancer and Infectious Diseases Center Komagome Hospital: Tokyo Toritsu Komagome Byoin

\section{Satoshi Morita}

Kyoto University Graduate School of Medicine Faculty of Medicine: Kyoto Daigaku Daigakuin Igaku Kenkyuka Igakubu

\section{Shinji Ohno}

Cancer Institute Hospital

Masakazu Toi

Kyoto University Graduate School of Medicine Faculty of Medicine: Kyoto Daigaku Daigakuin Igaku Kenkyuka Igakubu

Norikazu Masuda

NHO Osake National Hospital

\section{Research Article}

Keywords: Eribulin, Neoadjuvant chemotherapy, Sequential therapy, HER2-negative, locally advanced breast cancer

Posted Date: July 22nd, 2021

DOI: https://doi.org/10.21203/rs.3.rs-517868/v2

License: (c) (i) This work is licensed under a Creative Commons Attribution 4.0 International License. Read Full License 


\section{Abstract}

\section{Purpose}

The sequence of taxanes ( $T$ ) followed by anthracyclines (A) as neoadjuvant chemotherapy (NAC) has been the standard of care for almost 20 years for locally advanced breast cancer ( $L A B C)$. Sequential administration of eribulin (E) following $A / T$ could provide a greater response rate for women with $L A B C$.

\section{Methods}

In this single-arm, multicenter, Phase II prospective study, the patients received 4 cycles of the FEC regimen and 4 cycles of taxane. After the A/T-regimen, 4 cycles of $E$ were administered followed by surgical resection. The primary endpoint was the clinical response rate. Eligible patients were women aged 20 years or older, with histologically confirmed invasive breast cancer, clinical Stage IIIA (T2-3 and N2 only), Stage IIIB, and Stage IIIC, HER2-negative.

\section{Results}

A preplanned interim analysis aimed to validate the trial assumptions was conducted after treatment of 20 patients and demonstrated that clinical progressive disease (CPD) rates in the E phase were significantly higher (30\%) than assumed. Therefore, the Independent Data Monitoring Committee recommended stopping the study. Finally, 53 patients were enrolled, and 26 patients received the A/T/Eregimen. The overall observed clinical response rate (RR) was 73\% (19/26); RRs were 77\% (20/26) in the AT phase and $23 \%(6 / 26)$ in the E phase. Thirty percent (8/26) of patients had PD in the E phase, 6 of whom had achieved cCR/PR in the AT phase. Reported grade $\geq 3$ AEs related to E were neutropenia (42\%), white blood cell count decrease (27\%), febrile neutropenia (7.6\%), weight gain (3.8\%), and weight loss $(3.8 \%)$

\section{Conclusion}

Sequential administration of eribulin after the A/T-regimen provided no additional effect for LABC patients. Future research should continue to focus on identifying specific molecular biomarkers that can improve response rates.

\section{Introduction}

Neoadjuvant chemotherapy (NAC) for breast cancer has been used mainly for locally advanced cancer aiming at down-staging. The NSABP B-18 and B-27 trials showed similar overall survival (OS) and disease-free survival (DFS) rates between pre-operative and postoperative chemotherapy regimens $(1,2)$. Preoperative docetaxel administration increased the pathological complete response (pCR) rate by $12 \%$ $(2,3)$. In patients in whom pCR was obtained after NAC, both the OS and DFS rates were good (1). Currently, the $\mathrm{pCR}$ rate is used as an important parameter to assess the therapeutic effects of preoperative chemotherapy. As prognostic factors for $\mathrm{PCR}$ to preoperative chemotherapy, hormone 
receptor (HR)-negative and histological grade 3 have been reported $(4,5)$. However, no improvement in DFS or OS was seen, despite an improvement in the pCR rate. In NSABP B-27, AC and AC-DTX improved the $\mathrm{pCR}$ rate by $13 \%$, but there was no improvement in the prognosis (6). According to reviews of preoperative chemotherapy in Japan, the PCR rate for FEC administration after PTX therapy was $27.7 \%$, and the response rate was $85.1 \%$ (7). After FEC therapy, that for DTX administration was $25 \%$, and the response rate was $75 \%$ (8). Currently, as standard perioperative chemotherapy for breast cancer, sequential therapy with anthracycline and taxane preparations ( $A-T$ therapy) is performed; a more potent treatment has not been established. Recently, research on biomarkers to select patients for whom preoperative chemotherapy may be effective has been promoted. However, neither clinicopathological nor molecular biological markers to predict the tumor-reducing effects or long-term prognosis in preoperative chemotherapy for breast cancer have been sufficiently examined. Especially in those with a larger tumor diameter or more advanced cancer, it is difficult to achieve the disappearance of cancer cells using these drugs. Therefore, recently, as a new therapeutic strategy, A-T therapy simultaneously or sequentially combined with a new drug was proposed to improve clinical response and radical surgery rates. In the Gepar Trio Trial, the subjects were divided into 2 groups (responders and non-responders) after preoperative TAC therapy ( 2 courses), and the effects of additional treatment were examined. The responders were divided into 2 groups receiving TAC $\times 6$ (conventional arm) and TAC x 8 (responseguided arm), respectively. The sonographic response rates were $75.2 \%$ and $74.1 \%$, respectively. The physical response rates were $86.3 \%$ and $83.5 \%$, respectively (9). On the other hand, the non-responders were divided into 2 groups receiving TAC x 6 (conventional arm) and NX (response-guided arm), respectively. The sonographic response rates were $50.5 \%$ and $51.2 \%$, respectively (difference: 0.7 percentage points, $p=0.004)$. Those receiving NX showed significantly better results $(10)$. Recently, a report from the Gepar Trio Trial on long-term prognosis (SABCS2011) showed that NX (response-guided arm) prolonged DFS and OS more markedly than the conventional arm. In both the responders and the non-responders, the response-guided arm also showed better results. Furthermore, it improved the prognosis, with a DFS difference of approximately 10 percentage points after 5 years, whereas the difference in the response rate was 0.7 percentage points. In the Gepar Trio Trial, the establishment and concept of treatment differed from those in this study. However, these results suggest that, when the effects of standard treatment are not marked, another new regimen may improve the results of treatment. As described above, various efforts have been made to achieve better response in NAC for locally advanced breast cancer, Seung SJ et al. reported the 5-year survival rate for Stage III breast cancer is $70.2 \%$, indicating a poor prognosis (11). Therefore, in the case of inoperable locally advanced breast cancer, the standard chemotherapy regimens including anthracycline and taxane are currently not satisfactory.

Recently, the efficacy of a new microtubule inhibitor, eribulin, was evaluated in patients with breast cancer, and it was confirmed that eribulin significantly prolonged survival in AT-treated patients with advanced/recurrent breast cancer in comparison with conventional drugs selected by attending physicians. A phase II study of eribulin in Japan was conducted in subjects in whom the median number of previous chemotherapeutic regimens involving A and T preparations was 3 ( 1 to 5 ), of which 2 (0 to 3 ) 
were following progression/recurrence. The response rate was $21.3 \%$ (12). And Inoue et al. reported eribulin monotherapy was effective and well tolerated in heavily pretreated patients with metastatic breast cancer who had well-defined taxane resistance (13). Therefore, the results of standard chemotherapy with $A$ and $T$ preparations are not satisfactory in patients with locally advanced breast cancer $(L A B C)$ in whom surgery is impossible, and the tumor size may be more markedly reduced by sequentially combining eribulin with this therapy and thus prolonging survival.

The purpose of this study was to examine the clinical response rate of patients with advanced, local, HER2-negative breast cancer to sequential therapy with anthracycline, taxane, and eribulin. In addition, the histological effects, safety, and clinical usefulness were investigated. Clinical usefulness was evaluated using the proportion of patients undergoing radical surgery, the proportion of those undergoing breast-preserving surgery, relapse-free survival, and OS as parameters. In a sub-study, the relationship between the antitumor effects of eribulin and biological profiles of cancer tissue was examined using molecular biological and biochemical procedures.

\section{Methods}

\section{Patients}

Patients with LABC of stage IIIA (only T2-3/N2 are eligible), stage IIIB, and stage IIIC were recruited. All patients participating in this study had been histologically confirmed to have primary invasive adenocarcinoma of the breast with the tumor negative for HER-2 expression as determined by a local hospital laboratory (score of 0 or 1 on an immunohistochemical test [range, 0 to 3, with a score of 0 or 1 indicating HER2-negative breast cancer, a score of 2 a marginal result, and a score of 3 HER2-positive breast cancer; in the case of a marginal result, HER2 status was examined by means of fluorescence in situ hybridization to establish a positive or negative result] or a negative result on fluorescence in situ hybridization). Both protein and genetic status were estimated based on the guidelines for HER2 testing in breast cancer, as edited by American Society of Clinical Oncology/College of American Pathologists (14). Other key eligibility criteria were age of 20 to 70 years, Eastern Cooperative Oncology Group (ECOG) performance status score of 0 or 1 (on a 5-point scale, with higher numbers indicating greater disability), previously untreated (irradiation, chemotherapy, endocrine therapy, immunotherapy, or molecular targeted therapy) for $L A B C$, and patients who could provided written, informed consent.

Key exclusion criteria were the presence of breast cancer in bilateral breasts, other malignant conditions or synchronic multiple cancers, other concurrent serious disease that may interfere with planned treatment, including severe pulmonary conditions/illness, uncontrolled infections, uncontrolled diabetes, or uncontrolled hypertension, pregnant or lactating, hypersensitivity to any of the study medications, and chronic immunosuppressive therapies, including systemic corticosteroids.

\section{Trial design and oversight}


As preoperative therapy, eribulin therapy was performed after anthracycline/taxane (AT) therapy: sequential AT therapy with FEC100 (4 cycles) and taxane (4 cycles)(Figure 1). For anthracycline therapy, FEC100 was basically selected, but FEC75 or AC (60/600) was also possible, considering safety and age. For taxane therapy, DOC75 (4 cycles), weekly PTX 80 (4 cycles), or nab-PTX 260 (4 cycles) was used. The order of FEC100 and taxane administration was selected by the attending physicians. For eribulin therapy, 1 cycle consisted of 21 days. Eribulin at $1.4 \mathrm{mg} / \mathrm{m}^{2}$ was administered on Days 1 and 8 , and discontinued until Day 21. After the completion of the 4th cycle of eribulin therapy, antitumor effects were examined by surgery or tissue biopsy. When surgery was impossible, or when there was no evidence of adverse reactions affecting the continuation of administration in the absence of disease progression, the administration of eribulin was continued.

\section{End points}

The primary end point of the trial was the clinical response rate to sequential therapy with anthracycline, taxane, and eribulin in women with HER-2 negative LABC. Secondary end points included the pCR rate of the $A / T / E$ regimen, the safety of the $A / T / E$ regimen, $O S$ and $D F S$ of patients treated with the $A / T / E$ regimen, and the response rate to the $A / T / E$ regimen.

\section{Imaging modalities for determining treatment effect}

Imaging evaluation should be performed after sequential chemotherapy with anthracycline and taxane, and at the end of eribulin therapy. Imaging evaluation was performed using palpation, ultrasonography, CT or MRI, and the overall efficacy was determined based on RECIST ver1.1.

\section{Definition of pathological complete response (PCR)}

In this study, PCR was defined as no invasive residual in breast or nodes; noninvasive breast residuals allowed (ypTO/is ypNO).

\section{Statistical analysis}

The efficacy end point was the clinical response rate. According to a review on preoperative chemotherapy in Japan, Toi et al. reported the pCR was achieved in $25 \%$ of patients treated with DTX after FEC therapy. The response rate was $75 \%$ (15). A phase II study of eribulin in Japan was conducted in subjects in whom the median number of previous chemotherapeutic regimens involving the $A / T$ regimen was 3 ( 1 to 5 ), with a median of 2 ( 0 to 3 ) after progression or recurrence, and the response rate was $21.3 \%$. In the study by Toi et al mentioned above, the majority of the patients had T1 or T2 tumors (79.8\%), and $99.5 \%$ of patients were N0 or N1. However, our study included high-risk patients with stage IIIA (only T2-3/N2 are eligible), stage IIIB, and stage IIIC. Based on these points of view, it was anticipated that neoadjuvant treatment with eribulin following an A/T-regimen would produce a clinical response rate of at least $70 \%$, and a sample size of 47 evaluable patients was required (one-sided test of hypothesis; power of $80 \%$; alpha $=0.05$ ). Accrual of 60 patients was planned to allow for a $20 \%$ unevaluable rate. $A$ 
preplanned interim analysis that aimed to validate the trial assumptions was conducted after treatment of 20 patients, and it demonstrated that the CPD rate to the A/T/E regimen was significantly higher (30\%) than assumed.

\section{Results}

\section{Patients' characteristics}

The CONSORT diagram of the study is shown in Figure 2. A preplanned interim analysis that aimed to validate the trial assumptions was conducted after treatment of 20 patients, and it demonstrated that the CPD rate in the E phase was significantly higher (30\%) than assumed. Therefore, the Independent Data Monitoring Committee recommended stopping the study. A total of 53 patients were enrolled, and 26 patients received the A/T/E-regimen. The baseline demographic and clinical characteristics of the patients (27 patients of AT phase only and 26 patients of AT-E phase) are shown in Table 1 . For these 26 patients, the median age at diagnosis was 49 (32-67) years, 69\% (18/26) of the patients were hormone receptor (HR)-positive, and $31 \%(8 / 26)$ of the patients were HR-negative. The clinical tumor stage was T2 in 10 (39\%), T3 in 9 (35\%), and T4 in 7 (27\%). The clinical nodal status was N1 in 5 (19\%), N2 in 7 (27\%), and N3 in 14 (54\%). Clinical staging was as follows: Stage IIIA 23\% (6/26); Stage IIIB 23\% (6/26); and Stage IIIC 54\% (14/26).

\section{Clinical Response}

The clinical response rates (RRs) for each group are shown in Table 2. The overall clinical response was CR in 3 patients (11.5\%), PR in 13 patients (50.0\%), PD in 8 patients (30.8\%), and SD in 2 patients (7.7\%); the overall RR was $61.5 \%$ (16/26 patients).

In the AT phase, the RR was 76.9\% (20/26), with CR in 2 patients (7.7\%), PR in 18 patients (69.2\%), PD in 2 patients $(7.7 \%)$, and SD in 4 patients $(15.47 \%)$. In the E phase, the RR was $23.1 \%$, with CR in 3 patients (11.5\%) and PR in 3 patients (11.5\%); 30.8\% (8/26) of patients had progressive disease, with SD in 10 patients, (38.5\%) and N.E. in 2 patients (7.7\%).

The Waterfall plots are shown in Figure 3, showing change in tumor size after the initial anthracycline and taxane regimen from baseline (gray bar) and after completion of additional eribulin treatment from baseline (black bar) by RECIST v1.1 criteria.

\section{Pathological Response}

PCR was defined as no invasive residual in breast or nodes; noninvasive breast residuals allowed (ypT0/is ypN0). In 27 patients (28 lesions) who were treated with A-T alone, no pCR was obtained. Of the 26 patients who could be treated with A-T-E, pCR was observed in 4 of 25 patients except one patient who refused surgery, and the $\mathrm{pCR}$ rate was $16 \%(95 \% \mathrm{Cl}, 5.8-35.35)$.

\section{Adverse Events}


All 26 patients who received the A/T/E-regimen were included in the safety analysis (Table 3). In the A/T phase, the common hematologic adverse events were leukopenia, anemia, and neutropenia. The common non-hematologic adverse events in the A/T phase were fatigue, occurring in 20 patients $(76.9 \%)$, edema (73.1\%), peripheral sensory neuropathy (69.2\%), nausea (61.5\%), constipation (57.7\%), stomatitis (53.8\%), and nail change (53.8\%). Most adverse events were grade 1 or 2 in severity. In the A/T phase, neutropenia of grade 3 or 4 was noted in $34.6 \%$ of the patients, leukopenia of grade 3 or 4 in $38.5 \%$, and anemia of grade 3 or 4 in $38.5 \%$. Febrile neutropenia was noted in 2 patients $(7.7 \%)$.

In the $\mathrm{E}$ phase, the common hematologic adverse events were also leukopenia, anemia, and neutropenia. The common non-hematologic adverse events in the $E$ phase were fatigue, occurring in 18 patients (69.2\%), edema (65.4\%), peripheral sensory neuropathy (53.8\%), nail change (38.5\%), nausea (30.8\%), and constipation (26.9\%). In the E phase, neutropenia of grade 3 or 4 was noted in $38.5 \%$ of the patients, leukopenia of grade 3 or 4 in $50.0 \%$, and anemia of grade 3 or 4 in $34.6 \%$.

\section{Discussion}

The present results showed that there was no additional effect of sequential administration of eribulin following the anthracycline and taxane-regimen for patients with LABC. Although eribulin has clinical activity against metastatic breast cancer resistant to taxane, this study failed to demonstrate eribulin's clinical activity when given immediately subsequent to taxanes and anthracycline in the neoadjuvant setting. In metastatic breast cancer, eribulin is able to produce an equivalent PFS as first or second-line treatment compared with taxane (16). In contrast, administration of eribulin in the neoadjuvant setting showed less pathological response compared with taxane (17). These paradoxical activities of eribulin in different settings could account for the negative result seen in the present study.

Possible causes of the difference in the results of pre-operative eribulin treatment for early breast cancer and those of eribulin treatment for metastatic breast cancer include the following factors. First, eribulin has different modes of action: one is a blockade of cell cycle progression by inhibition of microtubule growth, and the other is non-mitotic complex effects on tumor biology, including induction of vascular remodeling, suppression of cancer cell migration and invasion, and reversal of the epithelial-tomesenchymal transition (18-24). Short-term exposure to eribulin of a bulky mass with stage III would not be enough to improve the tumor microenvironment. In terms of immediate tumor reduction, eribulin may be inferior to taxane. Eligible patients with stage III LABC require the maximum best response to obtain long-term survival. Other approaches to $L A B C$ should be investigated. However, in the adjuvant setting after tumor resection, a residual small tumor burden may allow activation of vascular remodeling by eribulin. A phase II trial of postoperative eribulin in breast cancer patients who did not achieve pCR following standard neoadjuvant chemotherapy showed that it was feasible and well tolerated (25). However, that study did not achieve its targeted 2-year DFS endpoints (improvements of $40 \%, 15 \%$, and $25 \%$ in TNBC, ER+, and HER2+, respectively) in any of the three cohorts treated, and the 2-year DFS in each cohort was similar to the result in the control arms of several large studies in which patients not achieving $\mathrm{pCR}$ received no subsequent adjuvant treatment. 
Second, there might be cross-tolerance between taxane and eribulin with immediate sequential exposure in a neoadjuvant setting for patients with LABC. In the EMBRACE Study (26), which examined patients who had undergone anthracycline- and taxane-based treatment for MBC, eribulin showed a 2.5-month extension of the median OS in patients who were taxane-refractory (median OS was 13.1 months for eribulin monotherapy and 10.6 months for TPC). However, Inoue et al. evaluated the clinical efficacy of eribulin in MBC patients who had well-defined taxane resistance, and they reported that the PD rate was $49.0 \%$ in a phase II, multicenter, single-arm, open-label clinical study (13).

The present study had a limitation. This study was planned to include both luminal and triple negative breast cancer patients. Seung SJ et al. reported the survival rates and median overall survival by breast cancer subtype. In their report, the 5 -year survival rate for stage III triple negative breast cancer is $47.2 \%$. Although the prognosis is $72.9 \%$ for the luminal type, it is not favorable (11). In HER2-positive breast cancer, based on the results of the APHINITY trial, sequential administration of docetaxel+trastuzumab+pertuzumab and anthracyclines is commonly used in locally advanced breast cancer, and a therapeutic strategy of sequential administration of T-DM1 has been developed as an additional treatment for patients who have failed to achieve PCR with preoperative chemotherapy. Under these circumstances, there is less possibility of developing sequential therapy with eribulin for HER2positive locally advanced breast cancer. As mentioned above, eribulin has shown efficacy in HER2negative metastatic breast cancer that is resistant to AT therapy, and eribulin could be an effective treatment option for high-risk locally advanced breast cancer with a poor prognosis. Since the position of eribulin in the HER2-positive and HER2-negative groups is different, it is important to conduct a study focusing on the HER2-negative group.

This study was conducted as an exploratory pilot study in a high-risk, locally advanced HER2-negative group with a poor prognosis. Depending on the results of this study, we could expect to explore the possibility of leading to further clinical trials targeting patient groups who might be expected to benefit from sequential administration of eribulin.

In summary, based on these features of eribulin and the present results, we do not recommend further development of sequential administration of eribulin following an anthracycline and taxane-regimen in the neoadjuvant setting for patients with LABC. Future research should continue to focus on identifying specific molecular biomarkers, which can improve response rates.

\section{Conclusion}

A preplanned interim analysis intended to validate the trial assumptions was conducted after treatment of 26 enrolled patients. The CPD rate after the eribulin-regimen was $30.8 \%$, and the pCR rate was $16 \%$. Toxicity was evaluated in 26 patients. The combination was safe, with mostly grade 1 and 2 toxicities. Because of the high CPD rate in response to the eribulin regimen, the study was terminated because of futility. Future research should continue to focus on identifying specific molecular biomarkers that can improve response rates. 


\section{Declarations}

\section{Funding;}

This work was sponsored by the Japan Breast Cancer Research Group (JBCRG) and operated in part using funds donated by pharmaceutical companies including Eisai Inc. This was an investigatororiginated and organized trial. Eisai Inc did not contribute to any part of the trial process, such as designbuilding or data management.

\section{Conflicts of interest/Competing interests;}

Yoshinori Ito received funding from AstraZeneca, Chugai, Daiichi Sankyo, MSD and Eli Lilly. Shoichiro Ohtani received remuneration from Chugai, Pfizer, AstraZeneca and Eli Lilly. Eriko Tokunaga received remuneration from Eli Lilly, Chugai and AstraZeneca, Consultant/ advisory role from Eli Lilly, Chugai and AstraZeneca. Masahiro Kashiwaba received remuneration from Chugai and Eli Lilly. Hidetaka Kawabata received funding from Taiho, Chugai, Novartis and Daiichi Sankyo. Katsumasa Kuroi received remuneration from Kyowa-Kirin, Eisai, and Chugai. Satoshi Morita received remuneration from AstraZeneca, Bristol Myers Squibb, Chugai, Eisai, Eli Lilly, MSD, Pfizer and Taiho. Shinji Ohno received remuneration from AstraZeneca, Eisai, Pfizer, Eli Lilly and Chugai, Funding from Eisai and Taiho. Masakazu Toi received remuneration from Chugai, Takeda, Pfizer, Kyowa-Kirin, Taiho, Eisai, DaiichiSankyo, AstraZeneca, Eli Lilly, Exact Science, Novartis, Shimadzu, Yakult, Nippon Kayaku, AFI technologies, Luxonus, Shionogi and GL Science, Consultant/advisory role from BMS, Daiichi-Sankyo, Eli Lilly, Terumo, Luxonus, Bertis, Kyowa-Kirin, Athenex Oncology and Kansai Medical Net, Funding from Chugai, Takeda, Pfizer, Kyowa-Kirin, Taiho, JBCRG association, Eisai, Daiichi-Sankyo, AstraZeneca, Astelas, Shimadzu, Yakult, Nippon Kayaku, AFI technologies, Luxonus, Shionogi and GL Science. Norikazu Masuda received remuneration from Chugai, AstraZeneca, Pfizer, Eli Lilly, Eisai and Takeda, Funding from Chugai, AstraZeneca, Kyowa-Kirin, MSD, Novartis, Pfizer, Eli Lilly, Eisai, Sanofi, Nippon Kayaku and Daiichi Sankyo. Ippei Fukada, Naoto Kondo, Masaya Hattori, Nobuki Matsunami, Kohjiro Mashino, Masahiko Tanabe, Taijiro Kosaka, Daisuke Yotsumoto, Kosho Yamanouchi and Masataka Sawaki declare that they have no conflict of interest.

Availability of data and material (data transparency); not applicable.

Code availability (software application or custom code); not applicable.

\section{Authors' contributions}

All authors contributed to the study conception and design. Material preparation, data collection and analysis were performed by Ippei Fukada, Yoshinori Ito and Norikazu Masuda. The first draft of the manuscript was written by Ippei Fukada and all authors commented on previous versions of the manuscript. All authors read and approved the final manuscript.

\section{Ethics approval}


All procedures performed in studies involving human participants were in accordance with the ethical standards of the FDA (ClinicalTrials.gov NCT01401959) and with the 1964 Helsinki declaration and its later amendments or comparable ethical standards.

Consent to participate (include appropriate statements);

Informed consent was obtained from all individual participants included in the study.

Consent for publication (include appropriate statements);

The authors affirm that human research participants provided informed consent for publication of the images in Figures 1, 2, and 3.

\section{Acknowledgments}

The authors would like to thank the patients who participated in the JBCRG-17 study and their families and the investigators and research coordinators at the participating institutions. The authors would also like to thank Nobuko Aoki, Michiro Soma, and their colleagues from the Japan Breast Cancer Research Group (JBCRG) administrative office for its contribution to the management of this study. In addition, the authors would like to thank the JBCRG data center for its contribution to patient registration, data management, and analyses.

Finally, the authors appreciate the efforts of Takashi Inamoto (Tenri Health Care University), Satoshi Teramukai (Department of Biostatistics, Graduate School of Medical Science, Kyoto Prefectural University of Medicine), Hajime Abe (Breast Center, Bellland General Hospital), and Akira Yoshioka (Department of Internal Medicine, Mitsubishi Kyoto Hospital) from the Independent Data Monitoring Committee.

\section{Informed consent}

Informed consent was obtained from all individual participants included in the study.

\section{References}

1) Wolmark N, Wang J, Mamounas E, Bryant J, Fisher B (2001) Preoperative chemotherapy in patients with operable breast cancer: nine-year results from National Surgical Adjuvant Breast and Bowel Project B-18. J Natl Cancer Inst Monogr. 30:96-102.

https://doi.org/10.1093/oxfordjournals.jncimonographs.a003469

2) Bear HD, Anderson S, Brown A, Smith R, Mamounas EP, Fisher B, Margolese R, Theoret $H$, Soran $A$, Wickerham DL, Wolmark N; National Surgical Adjuvant Breast and Bowel Project Protocol B-27 (2003) The effect on tumor response of adding sequential preoperative docetaxel to preoperative doxorubicin and cyclophosphamide: preliminary results from National Surgical Adjuvant Breast and Bowel Project Protocol B-27. J Clin Oncol. 21(22):4165-74. https://ascopubs.org/doi/10.1200/JC0.2003.12.005 
3) Symmans WF, Peintinger F, Hatzis C, Rajan R, Kuerer H, Valero V, Assad L, Poniecka A, Hennessy B, Green M, Buzdar AU, Singletary SE, Hortobagyi GN, Pusztai L (2007) Measurement of residual breast cancer burden to predict survival after neoadjuvant chemotherapy. J Clin Oncol. 25(28):4414-22. https://ascopubs.org/doi/10.1200/JC0.2007.10.6823

4) Ring AE, Smith IE, Ashley S, Fulford LG, Lakhani SR. Oestrogen receptor status, pathological complete response and prognosis in patients receiving neoadjuvant chemotherapy for early breast cancer (2004) $\mathrm{Br}$ J Cancer 91(12):2012-7. https://doi.org/10.1038/sj.bjc.6602235

5) von Minckwitz G, Raab G, Caputo A, Schütte M, Hilfrich J, Blohmer JU, Gerber B, Costa SD, Merkle E, Eidtmann H, Lampe D, Jackisch C, du Bois A, Kaufmann M (2005) Doxorubicin with cyclophosphamide followed by docetaxel every 21 days compared with doxorubicin and docetaxel every 14 days as preoperative treatment in operable breast cancer: the GEPARDUO study of the German Breast Group. J Clin Oncol. 23(12):2676-85. https://ascopubs.org/doi/10.1200/JC0.2005.05.078

6) Bear HD, Anderson S, Smith RE, Geyer CE Jr, Mamounas EP, Fisher B, Brown AM, Robidoux A, Margolese R, Kahlenberg MS, Paik S, Soran A, Wickerham DL, Wolmark N (2006) Sequential preoperative or postoperative docetaxel added to preoperative doxorubicin plus cyclophosphamide for operable breast cancer:National Surgical Adjuvant Breast and Bowel Project Protocol B-27. J Clin Oncol. 24(13):2019-27. https://ascopubs.org/doi/10.1200/JC0.2005.04.1665

7) Taguchi T, Masuda N, Nakayama T, Motomura K, Tsukamoto F, Shimazu K, Nomura T, Morimoto T, Yamamoto H, Wakita K, Nakano Y, Yoneda K, Inaji H, Takatsuka Y, Noguchi S; Kinki Breast Cancer Study Group (KBCSG) (2010) Phase II trial in Japan of sequential administration of weekly paclitaxel followed by FEC as neoadjuvant chemotherapy for locally advanced breast cancer [KBCSG0206 trial: Kinki Breast Cancer Study Group (KBCSG)]. Oncology. 78(5-6):302-8. https://doi.org/10.1159/000318169

8) Toi M, Nakamura S, Kuroi K, Iwata H, Ohno S, Masuda N, Kusama M, Yamazaki K, Hisamatsu K, Sato Y, Kashiwaba M, Kaise H, Kurosumi M, Tsuda H, Akiyama F, Ohashi Y, Takatsuka Y; Japan Breast Cancer Research Group (JBCRG) (2008) Phase II study of preoperative sequential FEC and docetaxel predicts of pathological response and disease free survival. Breast Cancer Res Treat. 110(3):531-9.

https://doi.org/10.1007/s10549-007-9744-z

9) von Minckwitz G, Kümmel S, Vogel P, Hanusch C, Eidtmann H, Hilfrich J, Gerber B, Huober J, Costa SD, Jackisch C, Loibl S, Mehta K, Kaufmann M; German Breast Group (2008) Intensified neoadjuvant chemotherapy in early-responding breast cancer: phase III randomized GeparTrio study. J Natl Cancer Inst. 100(8):552-62. https://doi.org/10.1093/jnci/djn089

10) von Minckwitz G, Kümmel S, Vogel P, Hanusch C, Eidtmann H, Hilfrich J, Gerber B, Huober J, Costa SD, Jackisch C, Loibl S, Mehta K, Kaufmann M; German Breast Group (2008) Neoadjuvant vinorelbinecapecitabine versus docetaxel-doxorubicin-cyclophosphamide in early nonresponsive breast cancer: 
phase III randomized GeparTrio trial. J Natl Cancer Inst.100(8):542-51.

https://doi.org/10.1093/jnci/djn085

11) Seung SJ, Traore AN, Pourmirza B, Fathers KE, Coombes M, Jerzak KJ. A population-based analysis of breast cancer incidence and survival by subtype in Ontario women. Curr Oncol. (2020) 27(2):e191e198. https://doi.org/10.3747/co.27.5769

12) Aogi K, Iwata H, Masuda N, Mukai H, Yoshida M, Rai Y, Taguchi K, Sasaki Y, Takashima S (2012) A phase II study of eribulin in Japanese patients with heavily pretreated metastatic breast cancer. Ann Oncol. 23(6):1441-8. https://doi.org/10.1093/annonc/mdr444

13) Inoue K, Saito T, Okubo K, Kimizuka K, Yamada H, Sakurai T, Ishizuna K, Hata S, Kai T, Kurosumi M (2016) Phase II clinical study of eribulin monotherapy in Japanese patients with metastatic breast cancer who had well-defined taxane resistance. Breast Cancer Res Treat (2016) 157(2):295-305. https://doi.org/10.1007/s10549-016-3808-x

14) Wolff AC, Hammond ME, Hicks DG, Dowsett M, McShane LM, Allison KH, Allred DC, Bartlett JM, Bilous M, Fitzgibbons P, Hanna W, Jenkins RB, Mangu PB, Paik S, Perez EA, Press MF, Spears PA, Vance GH, Viale G, Hayes DF; American Society of Clinical Oncology; College of American Pathologists (2014) Recommendations for human epidermal growth factor receptor 2 testing in breast cancer: American Society of Clinical Oncology/College of American Pathologists clinical practice guideline update. Arch Pathol Lab Med. 138(2):241-56. https://doi.org/10.5858/arpa.2013-0953-SA

15) Toi M, Nakamura S, Kuroi K, Iwata H, Ohno S, Masuda N, Kusama M, Yamazaki K, Hisamatsu K, Sato Y, Kashiwaba M, Kaise H, Kurosumi M, Tsuda H, Akiyama F, Ohashi Y, Takatsuka Y; Japan Breast Cancer Research Group (JBCRG) (2008) Phase II study of preoperative sequential FEC and docetaxel predicts of pathological response and disease free survival. Breast Cancer Res Treat.110(3):531-9.

https://doi.org/10.1007/s10549-007-9744-z

16) Minetta C. Liu, David W. Hillman, Ashley Elizabeth Frith, Alan P. Lyss, Douglas Jay Weckstein, Alvaro Moreno-Aspitia, Nguyet Le-Lindqwister, Claudine Isaacs, Donald B. Wender, DeQuincy Andrew Lewis, John T. Cole, Anthony John Jaslowski, Kendrith M. Rowland, Thomas H. Openshaw, Bret Edward Buckley Friday, Gini F. Fleming, Erik Asmus, Lauren J. Rogak, Ethan M. Basch, Amylou C. Dueck. Randomized phase III trial of eribulin (E) versus standard weekly paclitaxel $(P)$ as first- or second-line therapy for locally recurrent or metastatic breast cancer (MBC). JCO 38;15_suppl (ASC02020)

17) Abraham J, Robidoux A, Tan AR, Limentani S, Sturtz K, Shalaby I, Alcorn H, Buyse ME, Wolmark N, Jacobs SA (2015) Phase II randomized clinical trial evaluating neoadjuvant chemotherapy regimens with weekly paclitaxel or eribulin followed by doxorubicin and cyclophosphamide in women with locally advanced HER2-negative breast cancer: NSABP Foundation Study FB-9. Breast Cancer Res Treat. 152(2):399-405. https://doi.org/10.1007/s10549-015-3466-4 
18) Cadoo KA, Kaufman PA, Seidman AD, Chang C, Xing D, Traina TA (2018) Phase 2 Study of DoseDense Doxorubicin and Cyclophosphamide Followed by Eribulin Mesylate With or Without Prophylactic Growth Factor for Adjuvant Treatment of Early-Stage Human Epidermal Growth Factor Receptor 2Negative Breast Cancer. Clin Breast Cancer.18(6):433-440.e1. https://doi.org/10.1016/j.clbc.2018.04.001

19) Funahashi $Y$, Okamoto $K$, Adachi $Y$, Semba $T$, Uesugi $M$, Ozawa $Y$, Tohyama $O$, Uehara T, Kimura $T$, Watanabe H, Asano M, Kawano S, Tizon X, McCracken PJ, Matsui J, Aoshima K, Nomoto K, Oda Y (2014) Eribulin mesylate reduces tumor microenvironment abnormality by vascular remodeling in preclinical human breast cancer models. Cancer Sci. 105(10):1334-42. https://doi.org/10.1111/cas.12488

20) Gavert N, Ben-Ze'ev A. Epithelial-mesenchymal transition and the invasive potential of tumors. Trends Mol Med. 2008 May;14(5):199-209. https://doi.org/10.1016/j.molmed.2008.03.004

21) Polyak K, Weinberg RA (2009) Transitions between epithelial and mesenchymal states: acquisition of malignant and stem cell traits. Nat Rev Cancer. 9(4):265-73. https://doi.org/10.1038/nrc2620

22) Gunasinghe NP, Wells A, Thompson EW, Hugo HJ (2012) Mesenchymal-epithelial transition (MET) as a mechanism for metastatic colonisation in breast cancer. Cancer Metastasis Rev. 31(3-4):469-78. https://doi.org/10.1007/s10555-012-9377-5

23) Yoshida T, Ozawa Y, Kimura T, Sato Y, Kuznetsov G, Xu S, Uesugi M, Agoulnik S, Taylor N, Funahashi Y, Matsui J (2014) Eribulin mesilate suppresses experimental metastasis of breast cancer cells by reversing phenotype from epithelial-mesenchymal transition (EMT) to mesenchymal-epithelial transition (MET) states. Br J Cancer. 110(6):1497-505. https://doi.org/10.1038/bjc.2014.80

24) Dezső Z, Oestreicher J, Weaver A, Santiago S, Agoulnik S, Chow J, Oda Y, Funahashi Y (2014) Gene expression profiling reveals epithelial mesenchymal transition (EMT) genes can selectively differentiate eribulin sensitive breast cancer cells. PLoS One. 9(8):e106131.

https://doi.org/10.1371/journal.pone.0106131

25) Yardley DA, Peacock N, Daniel B, Anz B, Molthrop DC Jr, Shroff SK, Young R, Jankov A, Vander Woude A, Shastry M, Pasek J, DeBusk LM, Hainsworth JD (2020) Phase II trial of eribulin in patients who do not achieve pathologic complete response (pCR) following neoadjuvant chemotherapy. Breast Cancer Res Treat. 180(3):647-655. https://doi.org/10.1007/s10549-020-05563-z

26) Cortes J, O'Shaughnessy J, Loesch D, Blum JL, Vahdat LT, Petrakova K, Chollet P, Manikas A, Diéras V, Delozier T, Vladimirov V, Cardoso F, Koh H, Bougnoux P, Dutcus CE, Seegobin S, Mir D, Meneses N, Wanders J, Twelves C; EMBRACE (Eisai Metastatic Breast Cancer Study Assessing Physician's Choice Versus E7389) investigators (2011) Eribulin monotherapy versus treatment of physician's choice in patients with metastatic breast cancer (EMBRACE): a phase 3 open-label randomised study. Lancet. 377(9769):914-23. https://doi.org/10.1016/S0140-6736(11)60070-6 


\section{Tables}

Page 15/21 
Table 1

Patients' characteristics (AT phase only and AT-E phase)

\begin{tabular}{|c|c|c|c|c|}
\hline & \multicolumn{2}{|c|}{$\begin{array}{l}\text { AT phase only } \\
\text { ( } 27 \text { patients, } 28 \text { target lesion) }\end{array}$} & \multicolumn{2}{|c|}{$\begin{array}{l}\text { AT-E phase } \\
\text { ( } 26 \text { patients) }\end{array}$} \\
\hline & No. & $\%$ & No. & $\%$ \\
\hline \multicolumn{5}{|l|}{ Age (y) } \\
\hline $50>$ & 11 & 40.7 & 15 & 57.7 \\
\hline 50 and more & 16 & 59.3 & 11 & 42.3 \\
\hline \multicolumn{5}{|l|}{ Menopausal status } \\
\hline Premenopausal & 12 & 44,4 & 15 & 57.7 \\
\hline Postmenopausal & 15 & 55.6 & 11 & 42.3 \\
\hline \multicolumn{5}{|l|}{ PS } \\
\hline 0 & 26 & 96.3 & 25 & 96.2 \\
\hline 1 & 1 & 3.7 & 1 & 3.8 \\
\hline \multicolumn{5}{|l|}{ Clinical tumor size } \\
\hline $\mathrm{T} 2$ & 7 & 25.0 & 10 & 38.5 \\
\hline T3 & 8 & 28.6 & 9 & 34.6 \\
\hline T4 & 13 & 46.4 & 7 & 26.9 \\
\hline \multicolumn{5}{|c|}{ Clinical nodal stage } \\
\hline N1 & 6 & 21.4 & 5 & 19.2 \\
\hline N2 & 6 & 21.4 & 7 & 26.9 \\
\hline N3 & 16 & 57.1 & 14 & 53.8 \\
\hline \multicolumn{5}{|l|}{ Clinical stage } \\
\hline IIIA & 4 & 14.3 & 6 & 23.1 \\
\hline IIIB & 8 & 28.6 & 6 & 23.1 \\
\hline IIIC & 16 & 57.1 & 14 & 53.8 \\
\hline \multicolumn{5}{|l|}{ ER/PR status } \\
\hline ER+/PR+ & 13 & 46.6 & 13 & 50.0 \\
\hline $\mathrm{ER}+/ \mathrm{PR}-$ & 5 & 17.9 & 5 & 19.2 \\
\hline
\end{tabular}




\begin{tabular}{|llllc|}
\hline & $\begin{array}{l}\text { AT phase only } \\
\text { (27 patients, 28 target lesion) }\end{array}$ & $\begin{array}{l}\text { AT-E phase } \\
\text { (26 patients) }\end{array}$ \\
\hline Triple Negative & 10 & 35.7 & 8 & 30.8 \\
\hline
\end{tabular}

Table 2

Clinical response

\begin{tabular}{|c|c|c|c|}
\hline & Overall & AT phase & Eribulin phase \\
\hline \multicolumn{4}{|c|}{ All subtype $(n=26)$} \\
\hline CR & $3(11.5 \%)$ & $2(7.7 \%)$ & 3 (11.5\%) \\
\hline PR & $13(50.0 \%)$ & $18(69.2 \%)$ & $3(11.5 \%)$ \\
\hline PD & $8(30.8 \%)$ & $2(7.7 \%)$ & $8(30.8 \%)$ \\
\hline SD & $2(7.7 \%)$ & $4(15.4 \%)$ & $10(38.5 \%)$ \\
\hline NE & 0 & 0 & $2(7.7 \%)$ \\
\hline \multicolumn{4}{|c|}{ Luminal $(n=18)$} \\
\hline CR & $3(11.5 \%)$ & $2(7.7 \%)$ & $2(7.7 \%)$ \\
\hline PR & $8(30.8 \%)$ & $10(38.5)$ & $1(3.8 \%)$ \\
\hline PD & $1(3.8 \%)$ & $2(7.7 \%)$ & $3(11.5 \%)$ \\
\hline SD & $5(19.2 \%)$ & $3(11.5 \%)$ & $9(34.6 \%)$ \\
\hline NE & $2(7.7 \%)$ & $1(3.8 \%)$ & $3(11.5 \%)$ \\
\hline \multicolumn{4}{|c|}{ Triple negative $(n=8)$} \\
\hline CR & 0 & $1(3.8 \%)$ & 0 \\
\hline PR & $6(23.1)$ & $5(19.2 \%)$ & $2(7.7 \%)$ \\
\hline PD & $1(3.8 \%)$ & 0 & $3(11.5 \%)$ \\
\hline SD & 0 & $2(7.7 \%)$ & $2(7.7 \%)$ \\
\hline NE & $1(3.8 \%)$ & 0 & $1(3.8 \%)$ \\
\hline
\end{tabular}

$\mathrm{CR}$, complete response; PR, partial response; PD, progressive disease; SD, stable disease; $\mathrm{NE}$, not evaluable 
Table 3

Adverse events

\begin{tabular}{|c|c|c|c|c|}
\hline \multirow[t]{2}{*}{ Adverse event, $n$ (\%) } & \multicolumn{2}{|c|}{ AT phase $(n=26)$} & \multicolumn{2}{|c|}{ Eribulin phase $(n=26)$} \\
\hline & All grades & Grade $3 / 4$ & All grades & Grade $3 / 4$ \\
\hline \multicolumn{5}{|l|}{ Non-hematologic toxicities } \\
\hline Fever & $11(42)$ & 0 & $3(12)$ & 0 \\
\hline Fatigue & $20(77)$ & 0 & $18(69)$ & 0 \\
\hline Nausea & $16(62)$ & 0 & $8(31)$ & 0 \\
\hline Vomiting & $4(15)$ & 0 & 0 & 0 \\
\hline Anorexia & $6(23)$ & 0 & $4(15)$ & 0 \\
\hline Stomatitis & $14(54)$ & 0 & $3(12)$ & 0 \\
\hline Diarrhea & $11(42)$ & 0 & $3(12)$ & 0 \\
\hline Edema & $19(73)$ & 0 & $17(65)$ & 0 \\
\hline Constipation & $15(57)$ & 0 & $7(27)$ & 0 \\
\hline Arthralgia & $10(39)$ & 0 & $5(19)$ & 0 \\
\hline Myalgia & $10(39)$ & 0 & $6(23)$ & 0 \\
\hline Nail changes & $14(54)$ & 0 & $10(39)$ & 0 \\
\hline Weight loss & $1(4)$ & 0 & $5(19)$ & $1(4)$ \\
\hline Peripheral sensory neuropathy & $18(69)$ & 0 & $14(54)$ & 0 \\
\hline \multicolumn{5}{|l|}{ Hematologic toxicities } \\
\hline Neutropenia & $9(35)$ & $17(65)$ & $10(38)$ & $11(42)$ \\
\hline Leukopenia & $10(38)$ & $11(42)$ & $13(50)$ & $7(27)$ \\
\hline Anemia & $10(38)$ & 0 & $9(35)$ & 0 \\
\hline Febrile neutropenia & 0 & $2(8)$ & 0 & $2(8)$ \\
\hline
\end{tabular}

\section{Figures}




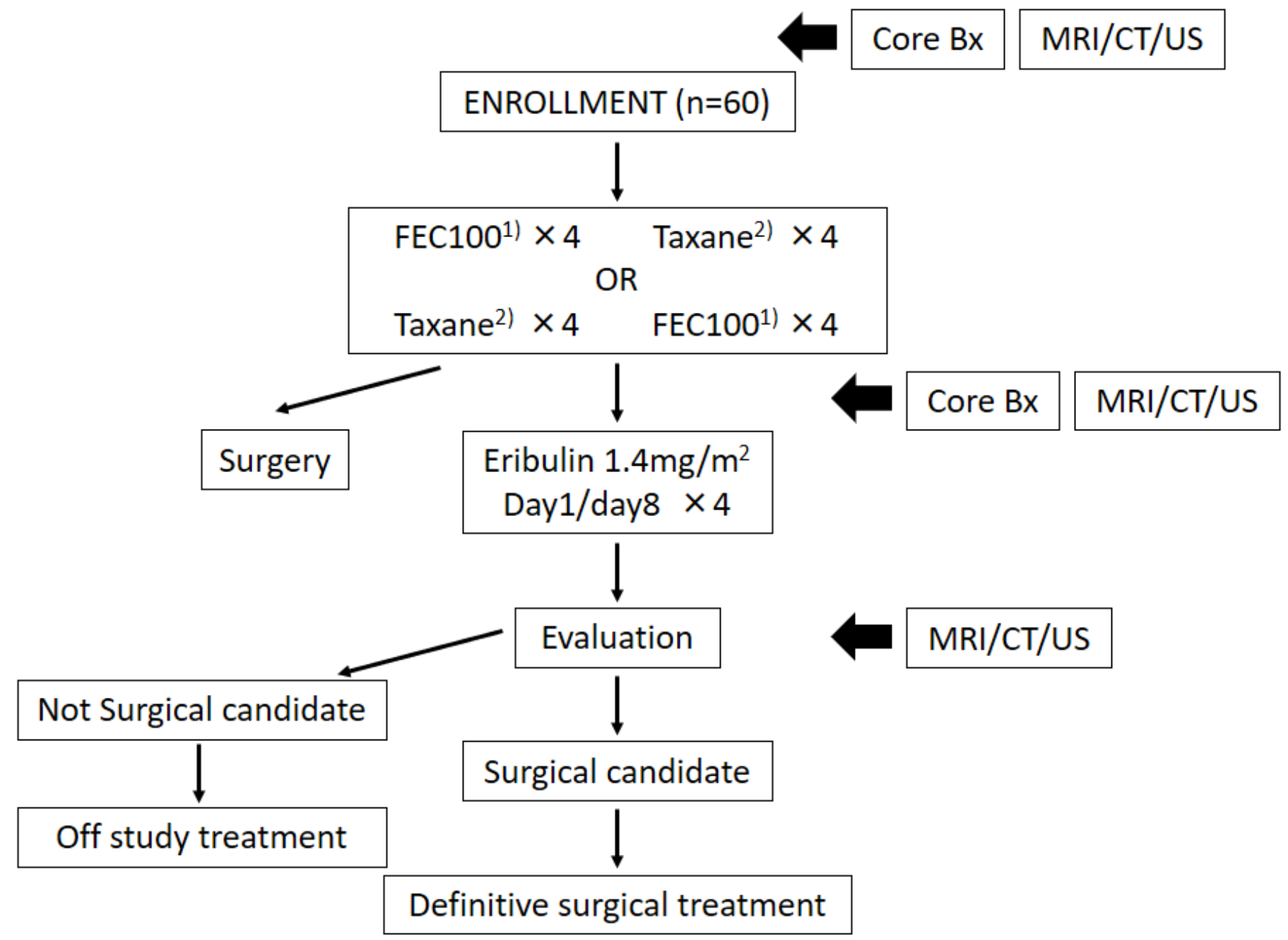

Figure 1

Study Design A single arm phase II study, preoperative sequential therapy with anthracycline and taxane followed by eribulin in patients with HER2-negative, locally advanced breast cancer. 1) FEC100; fluorouracil $500 \mathrm{mg} / \mathrm{m} 2$, epirubicin $100 \mathrm{mg} / \mathrm{m} 2$, and cyclophosphamide $500 \mathrm{mg} / \mathrm{m} 2$. 2) taxane therapy, Docetaxel $75 \mathrm{mg} / \mathrm{m} 2$, weekly Paclitaxel $80 \mathrm{mg} / \mathrm{m} 2$, or nab-Paclitaxel $260 \mathrm{mg} / \mathrm{m} 2$ was used. 


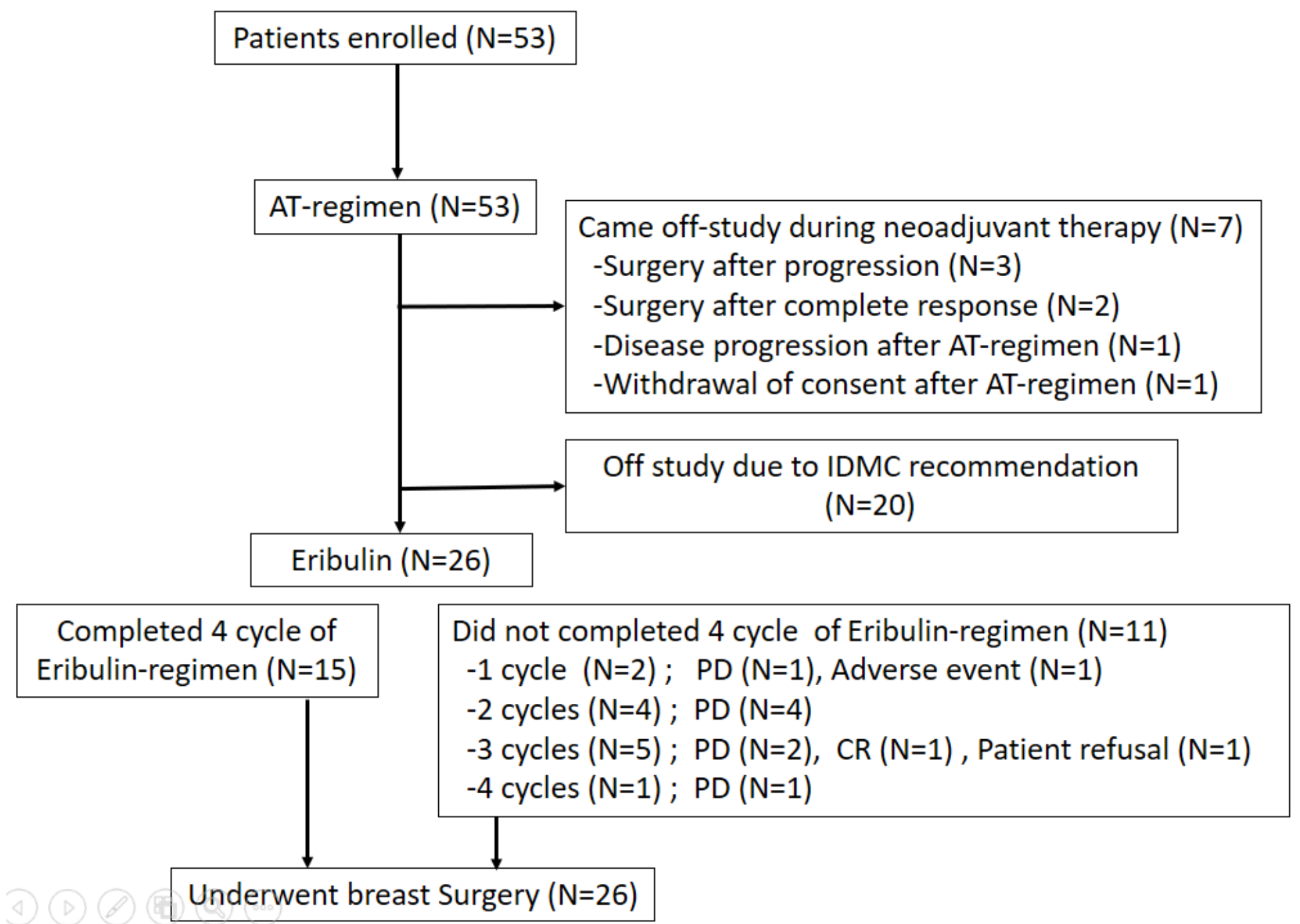

Figure 2

Study Consort Diagram A total of 53 patients were enrolled, 7patients were came off-study during neoadjuvant therapy and 20 patients were off study due to IDMC recommendation. Finally, 26 patients received the $A / T / E-r e g i m e n$, 


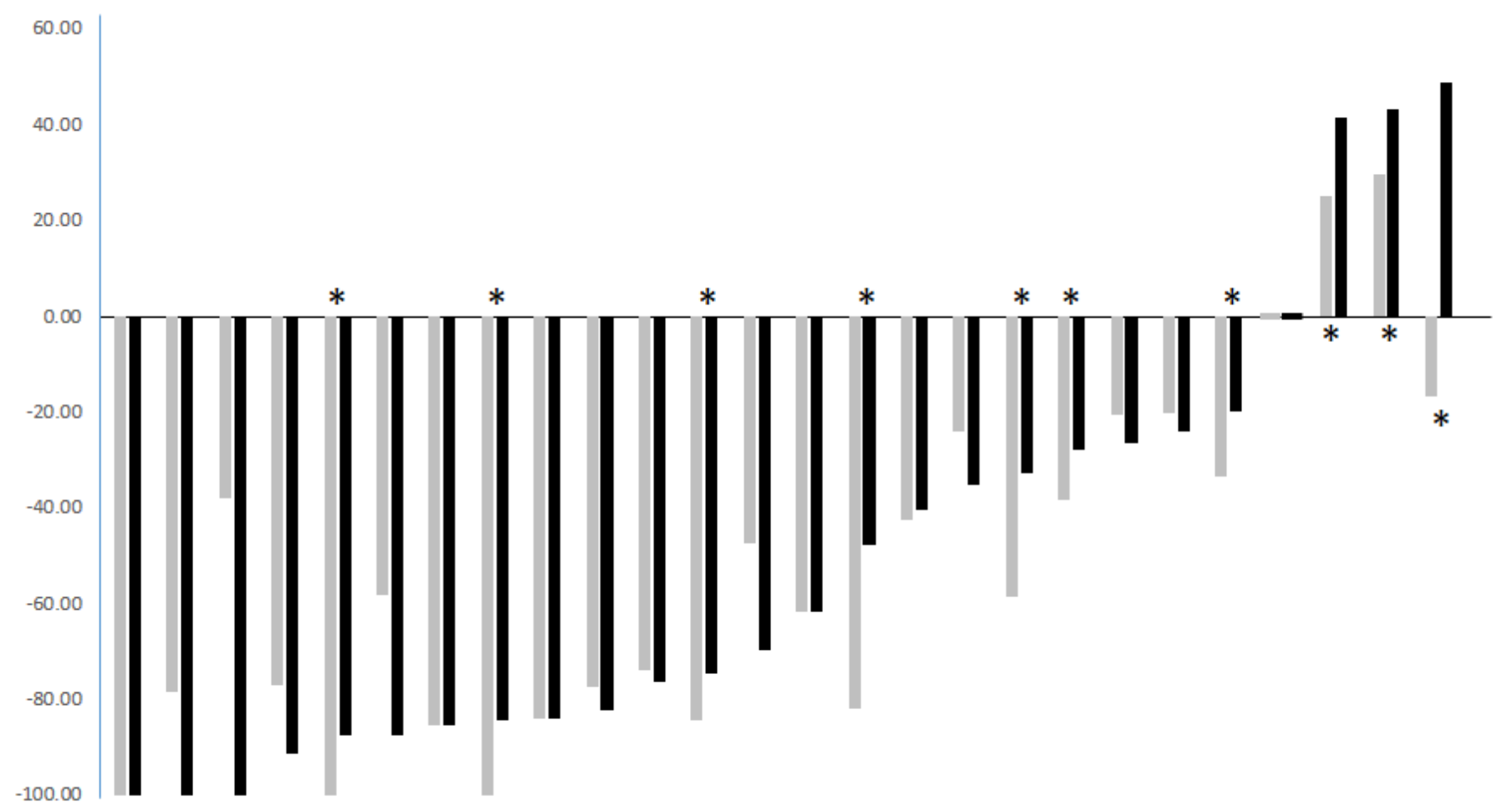

Figure 3

Waterfall plots of the percent change in tumor size The Waterfall plots showing change in tumor size after the initial anthracycline and taxane regimen from baseline (gray bar) and after completion of additional eribulin treatment from baseline (black bar) by RECIST v1.1 criteria. Asterisks ${ }^{*}$ ) indicate patients with disease progression during the periods of eribulin course. 